\title{
AN INTEGRAL EQUATION ARISING IN POTENTIAL THEORY ${ }^{1}$
}

\author{
H. T. JONES AND E. J. SPECHT
}

\begin{abstract}
This paper gives an integral equation, the solution of which is a solution of a classical problem in potential theory: Given a region with boundary $\mathscr{B}$, what distribution of charge on $\mathscr{B}$ will produce a potential having specified values on $\mathscr{B}$ ? The paper also indicates briefly how the integral equation is useful in simplifying certain proofs and extending certain theorems in potential theory.
\end{abstract}

The definitions and notation of this paper are those of [2, pp. 353-355]. Let $\alpha$ be a function having a derivative $D \alpha$ which satisfies a Hölder condition on $\mathscr{B}$. Let $\Psi$ be the potential due to the double-layer distribution whose moment-density is $\alpha$; that is, let

$$
\Psi z=\int_{0}^{s_{m}} \alpha s \cdot D_{u_{s}} \Gamma(\xi s-x, \eta s-y) d s \quad(z=x+i y),
$$

for each $z$ in the plane, except those on $\mathscr{B}$. Then [1, pp. 42-46] the normal derivative of $\Psi$ is continuous on $\mathscr{B}_{i} \cup \mathscr{B}$, where $\mathscr{B}_{i}$ denotes the union of the interior regions determined by $\mathscr{B}$. An expression for the limit of this normal derivative at the point $\zeta_{s}$ on $\mathscr{B}$ is the Cauchy principal value integral $\int_{\mathscr{I}}\left(D \alpha \cdot D_{1} \Lambda(s, \iota)\right)$, where $D_{1} \Lambda$ is the first-place partial derivative of $\Lambda$. The function whose value at $s$ is this integral will be denoted by $S \alpha$.

LEMMA 1. There exists a continuous function $\bar{\nu}_{1}$ such that $\lim _{z \rightarrow \zeta s} D_{u_{s}} \bar{\Phi}_{i} z=$ Sas, where $\bar{\Phi}_{i} z=\int_{\mathscr{I}}\left(\bar{v}_{1} \cdot \log (c /|\zeta-z|)\right)$ for all $z$ in $\mathscr{B}_{i}$.

Proof. The function $\bar{\nu}_{1}$ with the property stated exists if the equation $\bar{\nu}_{1}-T \bar{\nu}_{1}=-S \alpha / \pi$ has a continuous solution. Since the operator $T$ is compact in $\mathscr{L}^{2}\left[0, s_{m}\right]$, it follows by the Fredholm theory that this equation has a solution if and only if $\int_{\mathscr{I}}\left(S \alpha \cdot \psi_{j}\right)=0$ for $j=1, \cdots, m$, where $\psi_{1}, \cdots, \psi_{m}$

Received by the editors July 28,1971 .

AMS (MOS) subject classifications (1970). Primary 31A25, 31 A10.

Key words and phrases. Dirichlet problem, Neumann problem, integral equation, single-layer distribution, double-layer distribution.

${ }^{1}$ The major portion of the research reported in this paper was done while H. T. Jones was a participant in a program of Research Participation for College Teachers at the University of Oklahoma, supported by the National Science Foundation.

(c) American Mathematical Society 1973 
are $m$ linearly independent solutions of the equation $\psi_{j}-\bar{T} \psi_{j}=0$, the operator $\bar{T}$ being defined for each $\alpha$ in $\mathscr{P}$ by the equality $\bar{T} \alpha s=\int_{\mathscr{T}}(\alpha \cdot K(s, \iota))$. The functions $\psi_{1}, \cdots, \psi_{m}$ [2, equation (2)] defined by the relations

$$
\begin{aligned}
\psi_{j} s & =1 / c_{j} & & \text { if } \zeta_{s \in \mathscr{B}_{j},} \\
& =0 & & \text { if } \zeta_{s \in \mathscr{B}_{k}}, k \neq j
\end{aligned}
$$

are such solutions, so the conditions $\int_{\mathscr{I}}\left(S \alpha \cdot \psi_{j}\right)=0$ are equivalent to the conditions $\int_{s_{j-1}}^{s_{j}} S \alpha s d s=0$, which, by Green's Second Identity, are satisfied because $S \alpha$ is the normal derivative on $\mathscr{B}$ of a function harmonic in $\mathscr{B}_{i}$. Then every function of the form $\bar{\nu}_{1}+\sum_{j=1}^{m} a_{j} \varphi_{j}$, where $a_{1}, \cdots, a_{m}$ are numbers, is a solution of the equation $\gamma-T \gamma=-S \alpha / \pi$. Hence every function whose value at each point $z$ of $\mathscr{B}_{i}$ is $\int_{\mathscr{I}}\left(\left(\bar{v}_{1}+\sum_{j=1}^{m} a_{j} \varphi_{j}\right) \cdot \log (c /|\zeta-z|)\right)$ has the property that the limit of its normal derivative at $\zeta s$ is $S \alpha s$. That $\bar{\nu}_{1}$ is continuous on $\mathscr{B}$ follows from the form of the solution given by the Fredholm theory, when one takes into account property (iv), p. 362, of [2] and the fact that $S \alpha$ is continuous. Since every $\varphi_{j}$ satisfies a Hölder condition on $\mathscr{B}$, every solution given above is continuous on $\mathscr{B}$.

Lemma 2. There exists a continuous function $\nu_{1}$ such that $\Psi z=$ $\int_{\mathscr{I}}\left(v_{1} \cdot \log (c /|\zeta-z|)\right)$ for all $z$ in $\mathscr{B}_{i}$.

Proof. Let $\bar{\nu}_{1}$ be a particular function whose existence is assured by Lemma 1, and let $\bar{\Phi}_{i}$ be defined as in that lemma. Then $\lim _{z \rightarrow \zeta s} D_{u_{s}} \Psi z=$ $\lim _{z \rightarrow \zeta s} D_{u_{s}} \bar{\Phi}_{i} z$, and hence, by the uniqueness, to within an additive constant, of the solution of the interior Neumann problem, there exists a function $\Delta_{i}$ such that $\Psi=\bar{\Phi}_{i}+\Delta_{i}$ and, for some numbers $d_{1}, \cdots, d_{m}$, $\Delta_{i} z=d_{k}$ if $z$ is in the interior of $\mathscr{B}_{k}$, for $k=1, \cdots, m$. If $a_{1}, \cdots, a_{m}$ are chosen so that

then

$$
a_{j} \int_{\mathscr{I}}\left(\varphi_{j} \cdot \log (c /|\zeta-z|)\right)=-d_{j},
$$

$$
\Psi z=\int_{\mathscr{I}}\left(v_{1} \cdot \log (c /|\zeta-z|)\right)
$$

for all $z$ in $\mathscr{B}_{i}$, where $\nu_{1}=\bar{\nu}_{1}+\sum_{j=1}^{m} a_{j} \varphi_{j}$.

LEMMA 3. There exists a continuous function $v_{2}$ such that $\Phi_{e} z=\Psi z$ for all $z$ in the exterior, $\mathscr{B}_{e}$, of $\mathscr{B}$, where $\Phi_{\epsilon} z=\int_{\mathscr{I}}\left(v_{2} \cdot \log (c /|\zeta-z|)\right)$.

PRoof. There exists a function $v_{2}$ such that $\lim _{z \rightarrow \zeta s} D_{u_{s}} \Phi_{e} z=S \alpha s$ if the equation $v_{2}+T v_{2}=S \alpha / \pi$ has a solution. It does, in fact, have a unique solution $v_{2}$, as can be seen by noting that the equation $\gamma+\bar{T} \gamma=0$ has no nontrivial solutions and then applying the Fredholm theory in $\mathscr{L}^{2}\left[0, s_{m}\right]$. 
Hence, by the uniqueness, to within an additive constant, of the solution of the exterior Neumann problem, there is a constant function $\Delta_{e}$ such that $\Psi_{z}=\Phi_{e} z+\Delta_{e} z$ for all $z$ in $\mathscr{B}_{e}$.

Since $\Psi$ is a double-layer potential, $\lim _{\infty} \Psi=0$. Since the masses of $\Psi$ and $\Delta_{e}$ are both zero, the mass of $\Phi_{e}$ is zero, which implies that $\lim _{\infty} \Phi_{e}=0$ because $\Phi_{e}$ is a single-layer potential. Therefore $\lim _{\infty} \Delta_{e}=0$, so that $\Delta_{e}=0$, and hence $\Psi=\Phi_{e}$ on $\mathscr{B}_{e}$.

The continuity of $v_{2}$ can be established by a proof similar to that of the continuity of $v_{1}$ in Lemma 1 .

Lemma 4. If $\alpha, v_{1}$, and $v_{2}$ are defined as in the preceding lemmas, then

$$
2 \pi \bar{T} \alpha=L\left(v_{1}+v_{2}\right)
$$

and

$$
2 \pi \alpha=L\left(v_{1}-v_{2}\right),
$$

where $L$ is the operator defined for each $\gamma$ in $\mathscr{P}$ by $L \gamma t=\int_{\mathscr{I}}(\gamma \cdot \Lambda(\iota, t))$.

Proof. If $\Psi_{i}$ and $\Psi_{e}$ denote the restrictions of $\Psi$ to $\mathscr{B}_{i}$ and $\mathscr{B}_{e}$, respectively, then by Lemmas 2 and 3, and by the well-known boundary behavior of a double-layer potential,

and

$$
L v_{1} s=\lim _{z \rightarrow \zeta s} \Psi_{i} z=\pi \alpha s+\pi \bar{T} \alpha s
$$

$$
L v_{2} s=\lim _{z \rightarrow \zeta s} \Psi_{c} z=-\pi \alpha s+\pi \bar{T} \alpha s .
$$

Adding these equations gives (1) and subtracting them gives (2).

Lemma 5. If $\alpha, v_{1}$, and $v_{2}$ are defined as in the preceding lemmas, then

$$
v_{1}-v_{2}-T\left(v_{1}+v_{2}\right)=-(2 / \pi) S \alpha
$$

and

$$
T\left(v_{1}-v_{2}\right)=v_{1}+v_{2} .
$$

Proof. On the one hand, using the representations of $\Psi_{e}$ and $\Psi_{i}$ as single-layer potentials given in Lemmas 1 and 2, $\lim _{z \rightarrow \zeta s} D_{u_{s}} \Psi_{i} z=$ $-\pi v_{1} s+\pi T v_{1} s$ and $\lim _{z \rightarrow \zeta s} D_{u_{s}} \Psi_{e} z=\pi v_{2} s+\pi T v_{2} s$; on the other hand, using the definition of $\Psi$ as a double-layer potential, $\lim _{z \rightarrow \zeta s} D_{u_{s}} \Psi_{i} z=$ $\lim _{z \rightarrow \zeta s} D_{u_{s}} \Psi_{e} z=S \alpha s$. Adding the first two of these equalities and taking account of the third gives (3); subtracting the first two and taking account of the third gives (4). 
THEOREM 1. If $\alpha$ is a function such that $D \alpha$ satisfies a Hölder condition on $\mathscr{B}$, then there is a continuous function $\beta$ such that $\alpha=L \beta$ and

$$
\left(T^{2}-I\right) \beta=\left(1 / \pi^{2}\right) S \alpha .
$$

Proof. The existence of the function $\beta$ is given by Lemma 4 by taking $\beta$ to be $\left(v_{1}-v_{2}\right) / 2 \pi$. Operating on equation (4) with $T$ and using the result in equation (3) gives equation (5).

REMARK. Operating on equation (5) with $L$ and making use of the fact that $L T^{2}=\bar{T}^{2} L$ gives the equality $L S \alpha=\pi^{2}\left(\bar{T}^{2}-I\right) \alpha$, valid for all $\alpha$ satisfying the hypotheses of the theorem.

The following lemma, an extension of a result obtained by Kellogg [1, p. 46, footnote], gives an alternative characterization of the operator $S$.

LEMMA 6. If $\propto$ is a function such that $D \alpha$ satisfies a Hölder condition on $\mathscr{B}$, then $S \alpha=D L D \alpha$.

Proof. For each $s$ in $\mathscr{I}$ and each sufficiently small positive number $e$, let $\mathscr{E}=\{t:|A(s, t)|<e\}$. Since $D \propto$ and $D_{1} \Lambda(s, \iota)$ are continuous on $\mathscr{I}-\mathscr{E}$, it follows that

$$
D L D \alpha S=\lim _{e \rightarrow 0^{+}} \int_{\mathscr{I}-\mathscr{E}}\left(D \alpha \cdot D_{1} \Lambda(s, \iota)\right),
$$

provided that the limit exists and the convergence to the limit is uniform with respect to $s$.

To establish these facts, note that

$$
\begin{aligned}
\int_{\mathscr{I}-\mathscr{E}}\left(D \alpha \cdot D_{1} \Lambda(s, \iota)\right)= & \int_{\mathscr{I}-\mathscr{E}}(D \alpha t-D \alpha s)\left(D_{1} \Lambda(s, t)\right) d t \\
& +(D \alpha s) \int_{\mathscr{I}-\mathscr{E}} D_{1} \Lambda(s, t) d t .
\end{aligned}
$$

Since $D \propto$ satisfies a Hölder condition on $\mathscr{B}$ with exponent $d$, say, and since $D_{1} \Lambda$ is continuous on $\mathscr{I} \times \mathscr{I}$ except at points $(s, t)$ where $A(s, t)=0$, where it behaves like $|A|^{-1}$, it follows that the integrand of the first integral above behaves like $|A(s, t)|^{d-1}$, and hence the corresponding integral over $\mathscr{I}$ exists in the sense of Lebesgue. Moreover, the convergence of the first integral is uniform, being like that of $|A(s+e, s)|^{d}$. Now

$$
D_{1} \Lambda(s, t)=-\frac{X(s, t) D_{1} X(s, t)+Y(s, t) D_{1} Y(s, t)}{X^{2}(s, t)+Y^{2}(s, t)}+\frac{1}{A(s, t)},
$$

where $X$ and $Y$, defined on p. 363 of [2], have the properties that $X(s, \iota)$ and $Y(s, \iota)$ satisfy a Hölder condition on $\mathscr{B}$ with the same exponent $b$ as of that satisfied by $D \zeta$ and that $D_{1} X(s, \iota)$ and $D_{1} Y(s, \iota)$ are continuous except 
at points $t$ where $A(s, t)=0$, where they behave like $|A(s, \iota)|^{b-1}$. Therefore, for some $a>0$,

$$
\begin{aligned}
\left|\int_{\mathscr{S}-\mathscr{E}} D_{1} \Lambda(s, t) d t\right|< & a\left(|A(s, s-e)|^{b}+|A(s, s+e)|^{b}\right) \\
& +|\log | A(s, s-e)|-\log | A(s, s+e)||,
\end{aligned}
$$

from which both the existence of the limit and the uniform convergence for the second integral in (6) follow. This completes the proof of the lemma.

Theorem 1 and Lemma 6 can be used to give a more elegant proof of the existence and properties of the function $\Omega_{n}$ than was given in [2]. Finally, two other applications of Theorem 1 will be given in the next two theorems.

Lemma 7. If $\alpha$ and $\beta$ are functions as in Theorem 1 , then $\langle\beta, S \alpha\rangle=$ $-\|D \alpha\|^{2}$.

Proof. Since the function $D L D \alpha=S \alpha$ is continuous on $\mathscr{B}$,

$$
\langle\beta, S \alpha\rangle=\int_{\mathscr{I}}(\alpha \cdot D L D \alpha)=-\int_{\mathscr{I}}(D \alpha \cdot L D \alpha)=-\|D \alpha\|^{2} .
$$

THEOREM 2. Let $\mathscr{H}_{0}$ be the subspace of $\mathscr{H}$ which is orthogonal to the functions $\varphi_{1}, \cdots, \varphi_{m}$, let $\left\|T^{2}\right\|_{0}$ be the norm of $T^{2}$ on $\mathscr{H}_{0}$, let $\alpha$ be a function such that $D \alpha$ satisfies a Hölder condition on $\mathscr{B}$, let $\beta$ be the function such that $\alpha=L \beta$, whose existence is assured by Lemma 4 , let $\beta_{0}$ be the projection of $\beta$ on $\mathscr{H}_{0}$, and let $\beta_{1}=\beta-\beta_{0}$. Then

$$
\int_{\mathscr{I}} \alpha^{2} \leqq\|\alpha\|\left(\left\|\beta_{1}\right\|^{2}+\frac{\|D \alpha\|^{2}}{\pi^{2}\left(1-\left\|T^{2}\right\|_{0}\right)}\right)^{1 / 2} .
$$

Proof. From Theorem 1 and Lemma $7,\left\langle T^{2} \beta, \beta\right\rangle-\|\beta\|^{2}=-\|D \alpha\|^{2} / \pi^{2}$. Taking account of the facts that $\|\beta\|^{2}=\left\|\beta_{1}\right\|^{2}+\left\|\beta_{0}\right\|^{2}$, that $\left\langle T^{2} \beta_{0}, \beta_{1}\right\rangle=$ $\left\langle\beta_{0}, T^{2} \beta_{1}\right\rangle=0$, and that $T^{2} \beta_{1}=T^{2}\left(\sum_{j=1}^{m}\left\langle\beta, \varphi_{j}\right\rangle \varphi_{j}\right)=\sum_{j=1}^{m}\left\langle\beta, \varphi_{j}\right\rangle \varphi_{j}=\beta_{1}$, the above equality yields $\left\langle T^{2} \beta_{0}, \beta_{0}\right\rangle-\left\|\beta_{0}\right\|^{2}=-\|D \alpha\|^{2} / \pi^{2}$, or $\left\|\beta_{0}\right\|^{2} \leqq$ $\|D \alpha\|^{2} / \pi^{2}\left(1-\left\|T^{2}\right\|_{0}\right)$. Finally,

$$
\int_{\mathscr{I}} \alpha^{2}=\langle\alpha, \beta\rangle \leqq\|\alpha\| \cdot\|\beta\| \leqq\|\alpha\|\left(\left\|\beta_{1}\right\|^{2}+\|D \alpha\|^{2} / \pi^{2}\left(1-\left\|T^{2}\right\|_{0}\right)\right)^{1 / 2}
$$

The following result was obtained by Warschawski [3, p. 11] by using complex function theory. The proof given here would therefore be useful in extending his results to higher dimensions.

THEOREM 3. For $j=m+1, m+2, \cdots$, let $\varphi_{j}$ be the eigenfunction of $T$ associated with the eigenvalue $\lambda_{j}$, and let $\psi_{j}=L \varphi_{j}$. Then

$$
\left\|D \psi_{j}\right\|^{2}=\left(\pi^{2}\left(\lambda_{j}^{2}-1\right) / \lambda_{j}^{2}\right)\left\|\varphi_{j}\right\|^{2} .
$$


Proof. From equation (5) and Lemma 7 the result is obtained by noting that $\left\langle T^{2} \varphi_{j}, \varphi_{j}\right\rangle=\left\|\varphi_{j}\right\|^{2} / \lambda_{j}^{2}$.

\section{REFERENCES}

1. O. D. Kellogg, Potential functions on the boundary of their regions of definition, Trans. Amer. Math. Soc. 9 (1908), 39-50.

2. E. J. Specht and H. T. Jones, Compactness of the Neumann-Poincaré operator, Trans. Amer. Math. Soc. 140 (1969), 353-366.

3. S. E. Warschawski, On the solution of the Lichtenstein-Gershgorin integral equation in conformal maps, Nat. Bur. Standards Appl. Math. Ser., no. 42, U.S. Government Printing Office, Washingon, D.C., 1955. MR 17, 540.

Department of Mathematics, Andrews University, Berrien Springs, Michigan 49104 